\title{
Valproate: life-saving, life-changing
}

\author{
Author: Rhys $\mathrm{H}$ Thomas ${ }^{\mathrm{A}}$
}

Antiepileptic medications, and valproate principally, are commonly prescribed teratogens. There is significant concern that we are not doing enough to educate clinicians and potential parents about the risks of valproate in pregnancy. There is clear advice from the Medicines and Healthcare products Regulatory Agency and the International League Against Epilepsy about the risks of valproate exposure in utero. Reviews and guidelines that are focused on fetal risk, however, fall short in being able to fully replicate the complexity of a real clinical decision. Valproate is certainly life-changing if your child is one of the $10 \%$ with a major malformation or $30-40 \%$ with a neurodevelopmental disorder, but valproate is also potentially life-saving in the context of ensuring the best possible seizure control for some mothers with epilepsy. There are significant knowledge gaps regarding the risks to mothers who elect to take another drug, or to mother and baby if she comes off medication entirely. We also should be doing more to reduce rates of sudden unexpected death in epilepsy (SUDEP), which is recognised as a key target when evaluating all maternal deaths.

KEYWORDS: Autism, epilepsy, pregnancy, teratogens, valproate

\section{Introduction}

There is a common clinical paradox when selecting the correct treatment for a young woman with genetic generalised epilepsy. On the one hand, you can reassure her that about 4 in 5 people will become seizure free, but on the other hand you know that the drug most likely to bring this about has significant deleterious effects. Valproate is a drug that can be life transforming if it controls your epilepsy, but it is highly controversial because of the high rates of neurodevelopmental problems associated with children exposed to it in utero. This clinical conundrum is even more acute when all other drugs have failed to yield adequate seizure control and the only efficacious option that remains is valproate.

Thalidomide has become a touchstone for both the scientific and lay community when describing the potential scale of a drug side effect problem. Just alluding to the drug 'thalidomide' conjures up

Author: ${ }^{\text {A }}$ honorary consultant in epilepsy, Royal Victoria Infirmary and intermediate clinical lecturer, Newcastle University, Newcastle, UK both the widespread harm caused to an estimated 10,000 children worldwide and the battle to recognise this risk and restrict its use. The drug had (and still has) many important medical uses, but when taken in early pregnancy to combat hyperemesis, affected children were born with significant limb deficits. Although withdrawn in the UK in 1961, it took a long campaign and press support before there was a compensation settlement. Therefore, when The Daily Telegraph headline on 30 July 2009 stated that valproate was responsible for 'worst child poisoning case since thalidomide' the connotation was stark and emotional (Fig 1). I will discuss the many reasons why the thalidomide comparison is understandable, but also why the circumstances are significantly different. This will be with the specific aim of promoting the unanimous advice about valproate in pregnancy from many august agencies, while also recognising that there are important evidence gaps.

\section{Valproate exposure in utero}

Valproate is a recognised teratogen and the major cause of fetal anticonvulsant syndrome. An estimated $10.7 \%$ of children exposed to valproate in utero are born with a major congenital malformation (that is, a structural abnormality that is recognisable at birth) in comparison to an estimated $2 \%$ of unexposed infants. ${ }^{1}$ This produces the first two problems:

1 doctors are often poor at explaining risk

2 patients are inexperienced in balancing these risks.

This is exacerbated when the research evidence produces population risks ('a certain percent of affected children') whereas what we need is individualised risk ('your chance of having an affected baby is'). Equally, our prior experiences as physician and parent will sway the way we deal with perceived risk. The second issue is this: the fact that the concern regarding major malformations and valproate is so well known it has blinded us to the new scandal, the new relationship. We, as adult physicians have had 'change blindness' - we have failed to see that the threat of valproate is no longer limited to malformations at birth, but more far-reaching and life-changing developmental issues.

The easiest associations to recognise are when infrequent exposures (unusual drug $\mathrm{x}$ ) causes a dramatic, instant and frequently occurring outcome. A much more challenging situation is when a drug that has been available since the late 1960s, such as valproate, produces a delayed and variable effect, such as poorer cognition. Recognition is even harder when the effect is in the patients' offspring. This in part explains why the epilepsy community was so slow to recognise the extent of the risks. 
Fig 1. British newspapers respond to parental concern regarding valproate prescription in pregnancy.

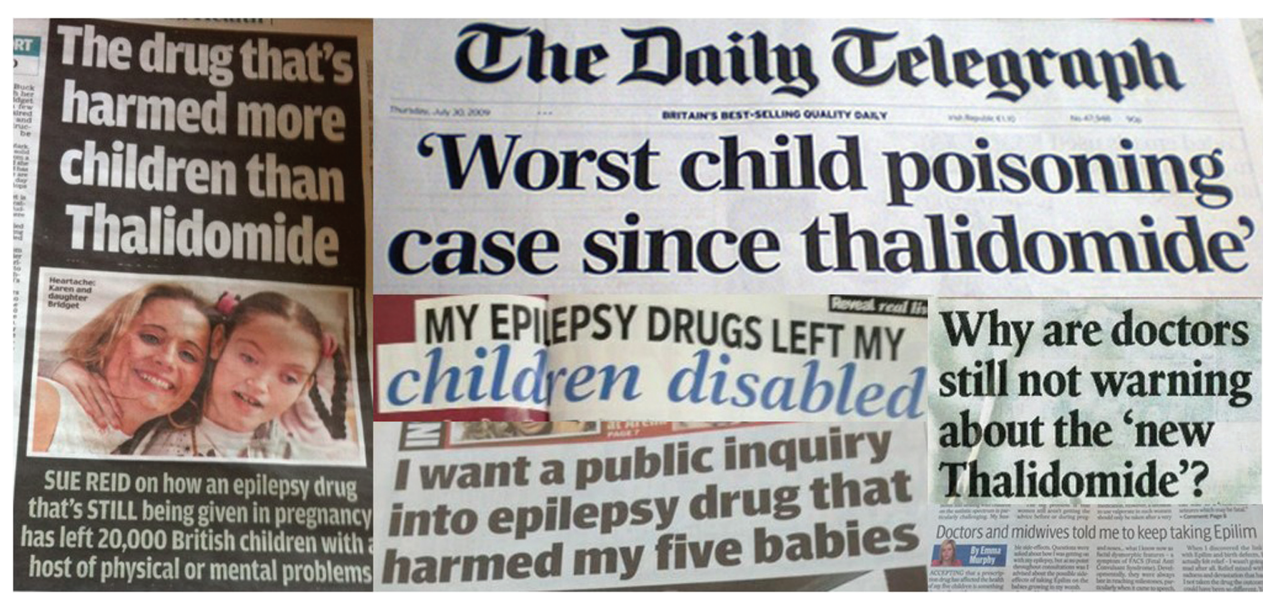

\section{The evidence}

The mainstay of evidence is epidemiological and as a result we can draw correlations but cannot speak directly to causation. These sources are either large retrospective cohort studies or they are smaller prospective studies. The pregnancy registries have been a great asset. Since 1996, the UK and Ireland Epilepsy and Pregnancy Register (www.epilepsyandpregnancy.co.uk) has been collecting information on women with epilepsy who became pregnant, with a view to determining the effect of antiepileptic drug exposure on the developing fetus. They currently have information on over 10,000 pregnancies and continue to collect around 800 new cases each year: about $25 \%$ of all eligible pregnancies. Typically, a mother with epilepsy will contact the register (or be invited to make contact) and complete a questionnaire about their health, their epilepsy and their medication, before the outcome of the pregnancy is known. Although there are undoubtedly biases about which types of women are motivated to participate and the ability of researchers to fully identify malformations or developmental issues without examining each child, the scale and rigour of the registries mean that they yield comparable results to each other.

The current concern is about adverse neurodevelopmental outcomes, intellectual impairment and autism. Prospective evidence that intellectual impairment could be identified in children as young as 3 came in 2009 as the study group published their landmark paper. ${ }^{2}$ At this stage, the threats of antiepileptic drugs were feared but not known - the relevant consensus guidelines by the American Academy of Neurology, ${ }^{3}$ and the International League against Epilepsy ${ }^{4}$ did not differentiate between the antiepileptic drugs. The authors identified 309 children exposed to antiepileptic drugs in utero; 258 were tested at 2 or 3 years using standardised age-appropriate tests. 53 were exposed to valproate, 73 to carbamazepine, 84 to lamotrigine and 48 to phenytoin; the mean IQ of the children exposed to valproate was 92 (CI 88-97), which is significantly lower than the IQs of the other groups of children (98 [CI 95-102], 101 [CI 98-104] and 99 [CI 94-104], respectively). This relationship was even more stark when the authors looked at smaller groups - eg high dose versus low dose: the 22 children exposed to high dose valproate had a mean IQ of 87. Higher maternal IQs were related to higher child IQs (as expected), except in the case of valproate. As expected, some children exposed to valproate had congenital malformations, and lower IQ - but these children alone did not explain the IQ differences. This relationship was confirmed when the study group were retested at 6 years. ${ }^{5}$ They helpfully demonstrated that breastfeeding did not increase the risks and conferred the expected benefits to infants' IQ. ${ }^{6}$

A Cochrane review of 22 observational prospective cohort studies and six register studies confirmed the observation of poorer neurodevelopmental outcomes of pregnancies exposed to valproate. ${ }^{7}$ The effect is dose related and exacerbated by polytherapy. In 2015, the European Registry of Antiepileptic Drugs and Pregnancy (EURAP) study group (an International registry of antiepileptic drugs and pregnancy) published on 1,224 pregnancies where valproate was taken alone and a further 364 where it was taken alongside another epilepsy drug. The rate of congenital malformation was $10 \%$ in children exposed to valproate $(11.3 \%$ if exposed to valproate and lamotrigine, $11.7 \%$ exposed to valproate and another antiepileptic drug that wasn't lamotrigine). However, the dose really mattered; when more than $1.5 \mathrm{~g}$ of valproate was used these rates increased to $24 \%$ for valproate alone, $31 \%$ for valproate and lamotrigine and $19.2 \%$ for valproate and another drug. ${ }^{8}$

In 2013, a Danish population-based study of 655,615 children born between 1996 and 2006 identified that mothers who took valproate in utero were five times more likely to have a child diagnosed with autism. This equates to a rate of 4.4/100 risk of autism spectrum disorder and a 2.5/100 risk of autism in childhood. ${ }^{9}$ Well-designed prospective studies have delineated the autistic traits in children. ${ }^{10}$ What is less clear - but potentially sobering - is the experimental work from rats which identify autism-like behaviours, induced by valproate, being transmitted to a third generation of pups, that themselves were not directly exposed to valproate. This finding has led some to speculate whether there are epigenetic processes that can lead to autism being passed on to the grandchildren of the mother who originally took the drug in pregnancy. ${ }^{11}$

\section{Advice}

In January 2015, the Medicines and Healthcare Products Regulatory Agency (UK) (MHRA) took the important step of issuing a statement regarding the safety of valproate when taken 
in pregnancy. ${ }^{12,13}$ This advice followed on from the European Medicines Agency in 2014 who recommended strengthening the restrictions on the use of valproate in women and girls. Physicians were quicker to release statements than governmental agencies; in 2009, the American Academy of Neurology recommended that valproate should be avoided in pregnancy whenever possible. ${ }^{14}$

The MHRA stated 'No-one should stop taking valproate without discussing it first with their doctor and the benefits of valproate treatment must be carefully balanced against the risks'. This message was echoed by the French health minister, Marisol Touraine, in early 2017, who announced practical measures such as adding an illustration to the verbal warning on the medicationpackaging and altering doctors' prescription software to include targeted information. The joint task force of the International League Against Epilepsy commission on European affairs and the European Academy of Neurology responded with a detailed letter. ${ }^{15}$ Their advice included that women of childbearing potential must use effective contraception during treatment. A consensus is evolving along the lines of the advice from the European Medicines Agency (Box 1).

The apparently contrary advice is that women should remain on medication throughout pregnancy. This oft repeated edict is based, primarily, on the risk of convulsions on the health of the fetus. Although this intuitively feels like the safest advice, it has never been studied with sufficient rigor and our evidence primarily comes from extrapolation from studies of eclampsia (where the pathophysiology of the seizures is very different) and from experiments on rats. Papers attempting to identify the risks of seizures on gestational outcome are all limited by the fact that there is often something inherently different about women with epilepsy who either seize frequently or need multiple drugs to control their epilepsy. A Thai study of 1,016 women with epilepsy identified that $49.5 \%$ had seizures in pregnancy. Women with epilepsy who had seizures during pregnancy were more likely to have lower educational achievement, be unmarried, have a lower family income and younger male partners. ${ }^{16}$ A study of 106 women with epilepsy on medication showed that babies of women who had one or more seizures in pregnancy were more likely to have a shorter gestational age and a fivefold higher risk of being born before term. ${ }^{17}$ However, the same study demonstrated

\section{Box 1.}

The Pharmacovigilance Risk Assessment Committee (PRAC) announced a pregnancy prevention programme in 2018, with six main points. ${ }^{43}$

Assess patients for the potential of becoming pregnant, and involve the patient in evaluating her individual circumstances and supporting informed decision making.

Pregnancy tests before starting and during treatment as needed.

Counsel patients about the risks of valproate treatment.

Explain the need for effective contraception throughout treatment.

Carrying out reviews of treatment by a specialist at least annually.

Introduce a new risk acknowledgement form that patients and prescribers will go through that there were drug risks too - women taking two or more antiepilepsy drugs were more likely to have small-for-gestationalage babies.

There is a compulsory medical birth registry in Norway and a study of 1,900 women with epilepsy ( $66 \%$ on no medication) identified that the majority of the poorer outcomes were seen in women who had epilepsy significant enough to need drug control. Both groups (medication versus no medication) exhibited seizures in the neonatal period. There were perinatal deaths in both groups ( $0.6 \%$ versus $0.7 \%)$; the difference between the two groups was non-significant. ${ }^{18}$ The advice that women should remain on their medication, therefore, does not equate that all women with epilepsy should be on medication in pregnancy. A UK study demonstrated that when pregnant, women with epilepsy were twice as likely to come off their medication compared with women before pregnancy - but should this have happened prior to conception? ${ }^{19}$ We are currently unable to tell which women would be safe to trial a withdrawal of medication when offering preconception counselling.

\section{Genetic generalised epilepsy}

The majority of mothers with epilepsy who take valproate have genetic generalised epilepsy; the evidence for the genetic component is compelling and there is an increased risk of epilepsy in first-degree relatives. The genetic architecture is complex and not yet fully elucidated. ${ }^{20,21}$ Clearly, the maternal 'epilepsy genes' plus any paternal contribution to risk can and will be transmitted to the infant. It is unknown whether parental genomic factors contribute towards valproate-associated neurodevelopmental disorders. Are women with genetic generalised epilepsy, therefore, at greater risk of neurodevelopmental disorders in their children than women with focal epilepsy? This is very hard to unpick because current practice is that valproate is the primary drug for genetic generalised epilepsy. Genetic generalised epilepsy is probably the only syndrome in which valproate could be justified in pregnancy because of our understating of the risks. Many pregnancy registers and epidemiological studies have been unable to report maternal epilepsy syndrome accurately. A study of 248 valproate-exposed pregnancies in 2008 identified that 126 had idiopathic generalised epilepsy and of these, 15 had a major malformation. They did not report on neurodevelopmental outcomes. Reports from other disorders are scanty - not least because women with migraine and bipolar disorder tend to come off medication before pregnancy. ${ }^{19}$

\section{Scale of the problem}

One of the reasons why valproate is a bigger issue than thalidomide is valproate is much more frequently prescribed in Europe and across the world. Women with epilepsy have $1 \%$ of all pregnancies and valproate has been widely prescribed since the late 1960 s. $^{22}$ It is estimated, therefore, that there are thousands of people in the UK living with iatrogenic valproate-associated neurodevelopmental disorders. Valproate remains a commonly prescribed drug and in 2002 was the most commonly prescribed antiepileptic drug worldwide. ${ }^{23}$ We know that there has been a decade-long reduction in valproate prescription in women aged $14-45$ years in Wales. ${ }^{24}$ In 2000/2001, valproate was the most prescribed drug to these women ( $45 \%$ ), falling to $24 \%$ by $2009 / 10$. Younger women ( 18 years and under) also saw valproate 
Fig 2. Total number of items of sodium valproate prescribed by GP practices across England from January 2012 to January 2017

Data available at www.OpenPrescribing.net, EBM DataLab, University of Oxford, 2017.

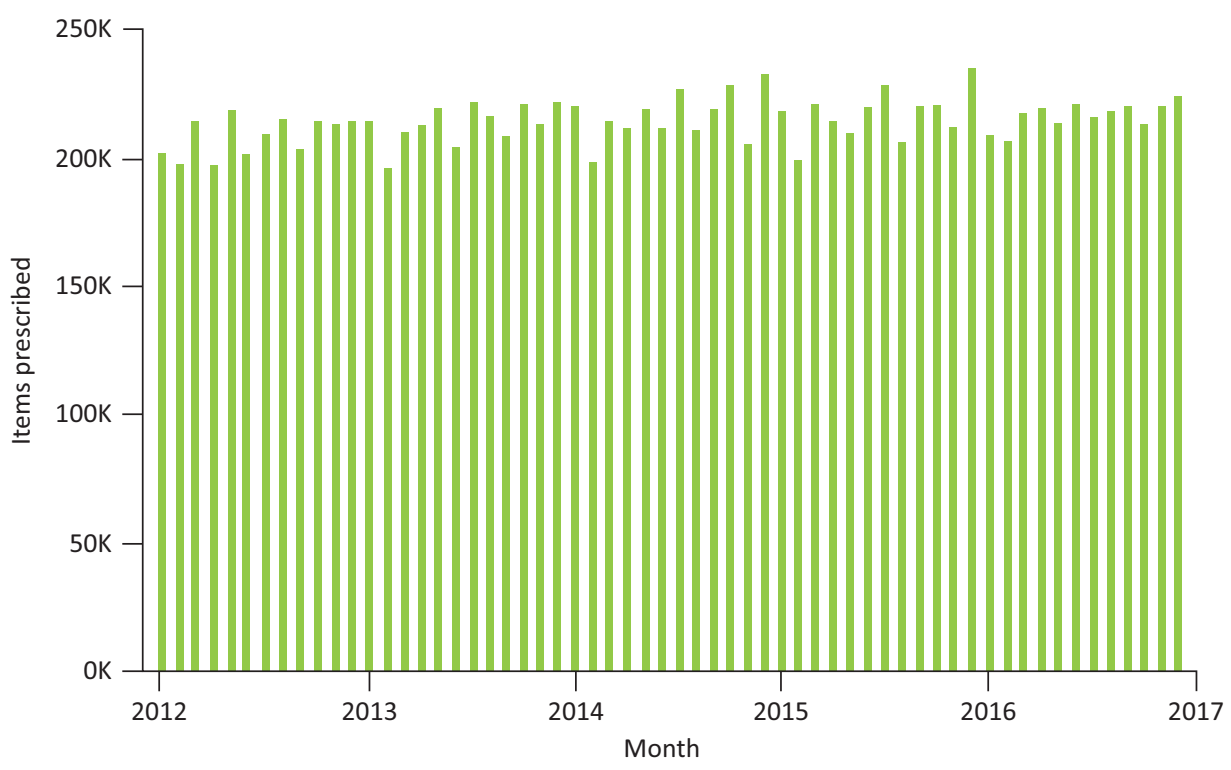

prescriptions fall from $74 \%$ to $46 \%$ during this period. Causing more concern however, current UK data (Fig 2) demonstrates that there has been no overall shift in the number of units of valproate that are being prescribed in primary care from 2012 to date. There is a significantly sized persistent group that remain on the drug despite strong evidence and clear advice, why might this be?

Fig 3 shows the variety in the prescription of valproate across clinical commissioning groups (CCGs) in England compared to all drugs for the control of epilepsy. CCGs like West Norfolk (far left) used 560 items of valproate out of 9,827 items for epilepsy in December 2016 compared with NHS Stockport (far right), which used 2,151 items of valproate out of 14,155 items for epilepsy control. This equates to a 2.6 -fold difference across the UK (57 versus 152 items of valproate per 1,000 items to control epilepsy). Does the presence of a strong epilepsy community as indicated by a local neurosciences centre influence valproate prescription (Fig 4)? Epilepsy prevalence is highly correlated with levels of deprivation; ${ }^{25}$ however, the map in Fig 4 is not a 'deprivation
Fig 3. Proportion of valproate items prescribed compared to all items prescribed for control of epilepsy. All CCGs are shown on the graph online, available at www. OpenPrescribing.net, EBM DataLab, University of Oxford, 2017.

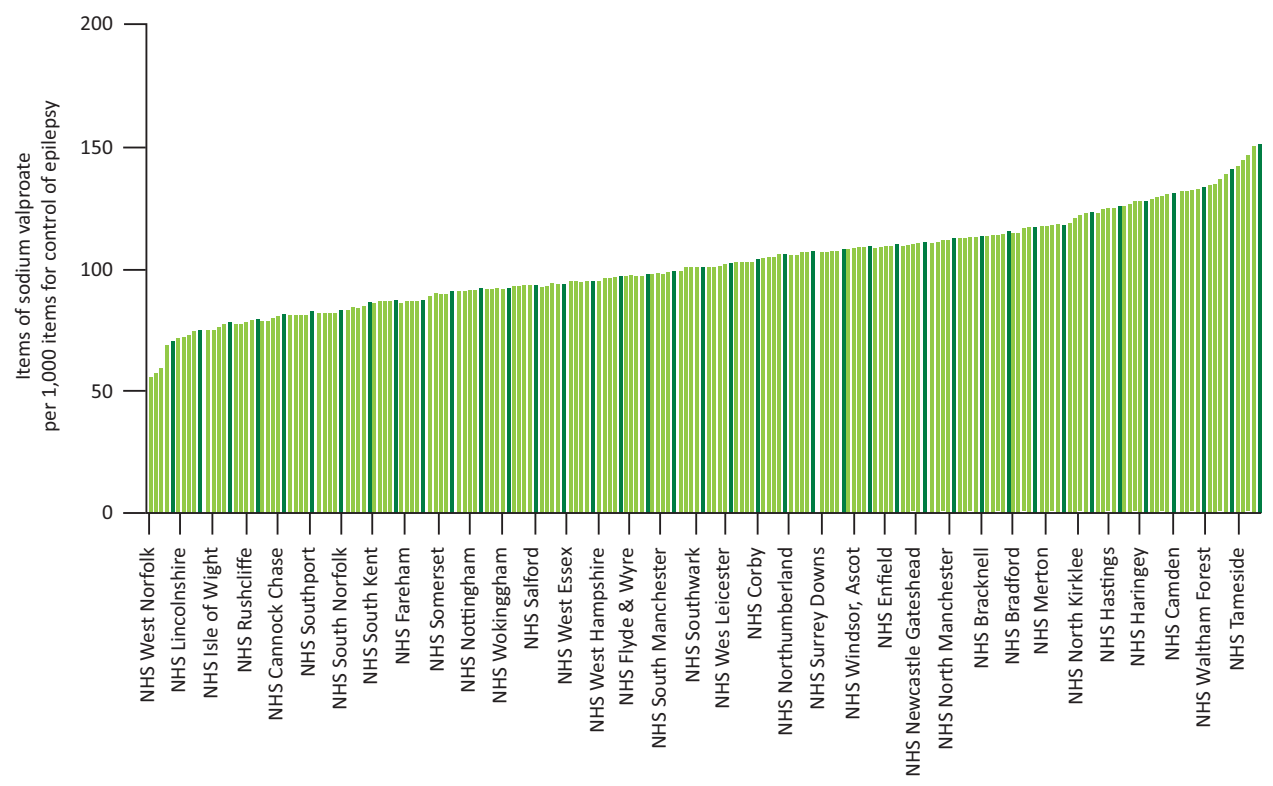

Clinical commissioning group 


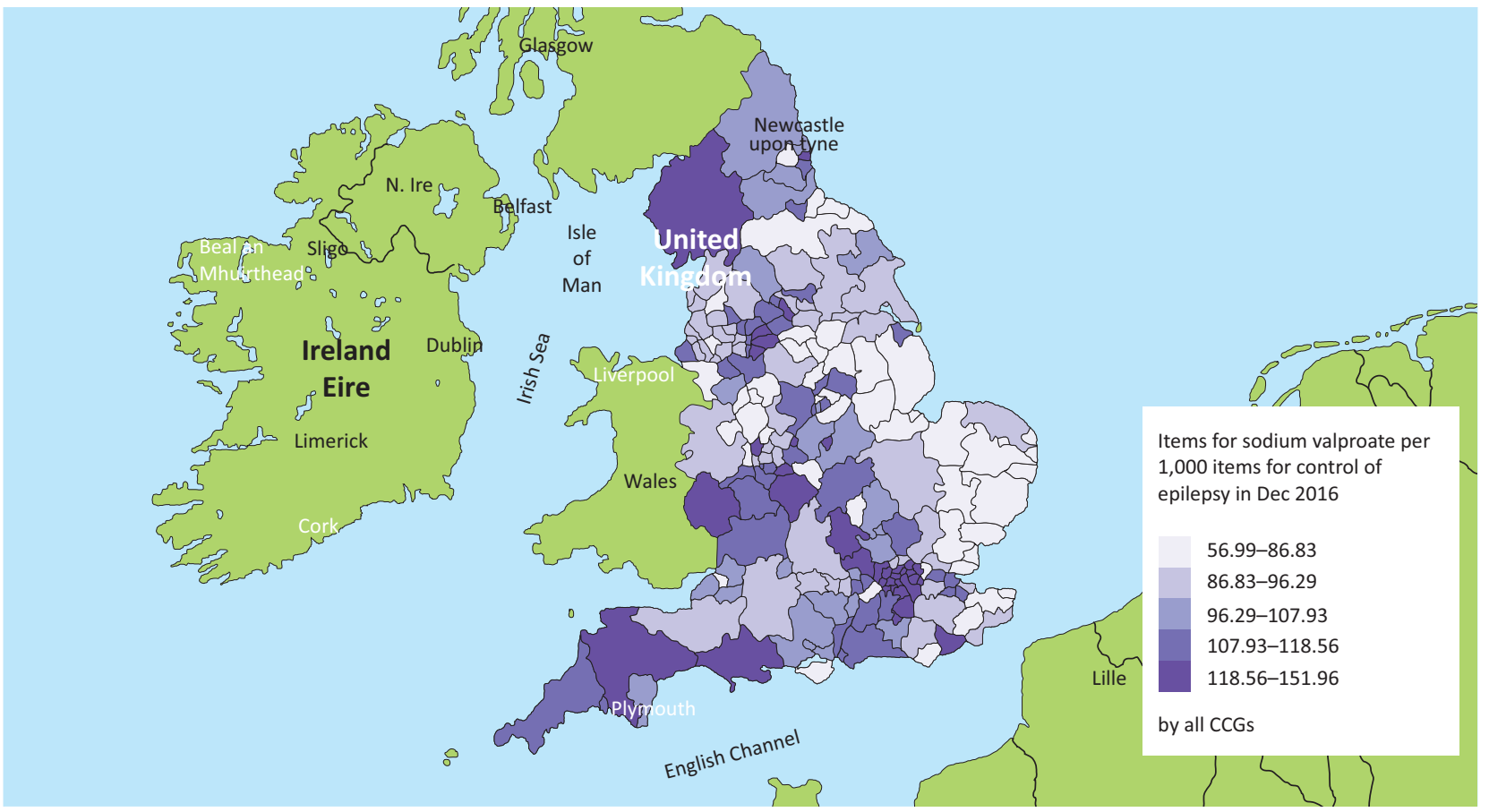

Fig 4. Geographical variability of valproate prescribing in England, compared to all items for epilepsy. There appears to be an east versus west divide. Some clinical commissioning group areas of higher valproate prescription do not have a neurosciences centre: NHS Cumbria, Herefordshire, South Tyneside, North Kirklees. There is a 'M40-M25' corridor of high prescription around Oxford and north and west London. The map uses the data from Fig 3, also available at www.OpenPrescribing.net, EBM DataLab, University of Oxford, 2017.

map' of England nor does it correspond to increased epilepsy prevalence. CCGs in the top $20 \%$ should embrace the challenge of reducing their valproate prescribing and learn how this is possible from the best performing CCGs.

\section{Valproate is an effective drug}

One of the main reasons that valproate is not like thalidomide is that we cannot simply stop prescribing it completely. Influential, UK-based, pragmatic clinical trials such as SANAD (Standard And New Antiepileptic Drugs) reinforce the clinical view that valproate is superior to many of the subsequent antiepileptic drugs and is the most efficacious in genetic generalised epilepsy. ${ }^{26} \mathrm{~A}$ total of 716 patients were randomised to open-label lamotrigine, topiramate or valproate. Valproate was superior in terms of time to treatment failure (a compound metric of 'do people remain on the drug?' combining both side effects and seizure control). Valproate's strength was primarily in seizure control.

Seizure control is not the only thing important to potential mothers with epilepsy - but it is important. Most quality of life outcomes correlate with seizure control; sleep, mood, anxiety, confidence, pain; all are worse when seizures are frequent. UK driving restrictions apply to almost all people with recurrent seizures, meaning that a mother with an absence seizure following a period of control must surrender her licence for at least a year before a return to driving. This clearly has a negative consequence on employment and employability despite legislation to protect against overt discrimination.

Furthermore, seizures themselves are not benign. The MBRRACEUK (Mothers and Babies: Reducing Risk through Audits and
Confidential Enquiries across the UK) report in December 2014 recognised that 'epilepsy remains a high risk condition in pregnancy' continuing 'multiagency evidence-based guidelines are urgently required to standardise and improve the care of pregnant and postpartum women with epilepsy'. Epilepsy was a comorbidity in 17 (7.8\%) maternal deaths in pregnancy. Of the women who died in pregnancy, not one was reported as taking valproate. Lamotrigine was the most commonly prescribed drug; the pharmacodynamics of lamotrigine mean that some clinicians regularly test levels in pregnancy, whereas many do not. ${ }^{27}$ In total, 14 deaths are reported on in detail - all caused by poor seizure control; 12 were SUDEP (sudden unexpected death in epilepsy) and two were drownings. ${ }^{28}$ Only two of these twelve who died from SUDEP had adequate seizure control prior to pregnancy; poor seizure control correlates with an increase in SUDEP risk. Furthermore, a review of similar deaths over 30 years concluded that between 1979 and 2008, the proportion related to epilepsy have increased although maternal deaths have decreased. ${ }^{29}$ Put bluntly, restricting efficacious drugs from women increases their risk of dying from their epilepsy. Even when a woman is not pregnant, epilepsy confers a twentyfold increase in death because of SUDEP. SUDEP kills more Britons per year than asthma or AIDS or house fires. Hence, when the International League Against Epilepsy issued their report in 2015, there were authors rebutting their advice with letters such as 'valproate still has a place in women with epilepsy'. ${ }^{30}$

\section{Valproate the drug}

Valproate was not the output of any rational drug discovery programme, rather a serendipitous accident. Pierre Eymard was 
performing experiments on rat models of epilepsy in France in the early 1960s. Valproic acid was used as a lipophilic vehicle for compounds that were water insoluble. Each compound dissolved in the valproic acid was a very acceptable antiepileptic agent, but so too was the control vehicle with no 'active' compound in it. Very quickly they moved to use sodium valproate in rabbits and then other groups performed the first in-man studies in 1964. By 1967, it was marketed in France as Depakine - within 5 years of its discovery. ${ }^{31}$

Valproic acid has several formulations and many indications, including migraine prophylaxis, mood stabilisation and, to a lesser degree, pain control. This means that advice such as that given by the MHRA needs to be advertised widely beyond the narrow scope of neurology in secondary care. Valproic acid is theorised to have many modes of action:

$>$ inhibition of GABA transaminase

> suppression of repetitive neuronal firing through inhibition of voltage-sensitive sodium channels

$>$ histone deacetylase inhibitor function.

The combination of a 'happy accident' discovery and a varied mode of action has stymied researchers as they have sought to identify what is it specifically about the molecule that confers the risk in pregnancy as they aim to design safer valproates.

There are a number of possible and potentially overlapping explanations for the association between exposure to valproate in utero and neurodevelopmental disorders in children. These include the following.

> There is a direct effect on brain development.

> The association is genetic but pleiotropic, driven by genetic overlap between the maternal epilepsy and causes of neurodevelopmental disorder.

> There is a 'gene $x$ environment' interaction and a high genetic risk of genetic generalised epilepsy from the mother renders the developing child more susceptible to valproate's effects on brain development.

$>$ Valproate is potentially mutagenic and there is an excess of de novo mutation in the children exposed to valproate.

What is telling is that one of the methods of modelling 'autism' in rats is to expose them to high doses of valproate in utero. ${ }^{32}$ Rat models provide a better method of studying the direct brain effects of valproate. ${ }^{33}$ Initially, exposed rat pups show alteration in medial prefrontal cortex synaptic connectivity. Their principal neurons show decreased excitability but increased local connectivity, due in part to an up-regulation of NMDA receptor expression. The synaptic abnormalities in exposed rats persist into adulthood.

Valproate is primarily used in the epilepsy syndrome genetic generalised epilepsy (synonymous with idiopathic generalised epilepsy). ${ }^{26}$ This is a developmental epilepsy syndrome that often starts in childhood or teenage years and can be exacerbated by sleep deprivation and alcohol. There are also neurocognitive problems associated, including some executive function difficulties and impulsivity problems. ${ }^{34,35}$ This is partly why the cognitive outcomes in the offspring of this group were not recognised; in part maternal under-reporting and in part a paternalistic doctorly view that these borderline IQs may be commensurate with maternal abilities. However, in fairness, it is worth adding that experts were asking questions about the safety of epilepsy drugs in pregnancy as early as 1964 - prior to the introduction of valproate. ${ }^{36}$ What we have not proven with sufficiently powered studies is the role that maternal epilepsy syndrome plays on the likelihood of neurodevelopmental outcomes in the child. ${ }^{37}$ The reasons why this is crucial are laid out below.

\section{Epilepsy is different}

In 2011, Phil Smith and I wrote that 'epilepsy is different'; ${ }^{38}$ this editorial was a call to re-examine the series of contradictions that surround epilepsy - such as it is potentially invisible, yet stigmatising; controllable, yet a cause of sudden death; common, but so little is known about it by lay audiences. Another contradiction is that family studies and twin registries tell us that it should be highly heritable and yet we know surprisingly little about the genetic architecture of common epilepsies. ${ }^{20}$ That transmissible genetic factors are important is incontrovertible and a recent paper identifies for the first time that these mutations are also those that are seen to cause severe childhood epilepsies with autism and intellectual disability. ${ }^{39}$

There are several important research questions that need to be answered.

1 Why are some children preferentially affected and can this be determined by studying mothers (ideally prior to conception)? Could we identify 'high-risk' and 'low-risk' women to personalise preconception counselling?

2 Is valproate the only drug that is working in this way? Are there other drugs working via similar mechanisms that have a similar result but to a lesser degree and so are yet to be discovered?

3 With the understanding now about very rare gene changes in people with common epilepsies, what role does the maternal epilepsy syndrome have on the child? Do women with migraine and bipolar disorder have similar problems?

4 Does valproate cause new mutations in the child? We know that these de novo mutations are an important cause of intellectual disability and autism. Approximately $40 \%$ of children with intellectual disability have a causative de novo mutation identifiable with genome sequencing. ${ }^{39,40}$ The mechanisms by which any teratogen affects organogenesis are still unclear but there is a large overlap between mutagenicity and teratogenicity. As the evidence that valproate is a teratogen is indisputable, we have to consider whether it is mutagenic. ${ }^{41}$ Valproate readily crosses the placenta and circulates in the embryo in higher concentrations than in maternal blood.

\section{Conclusions}

It is clear from consensus statements and an independent review of the evidence that valproate confers a risk of neurodevelopmental disorder in exposed infants. This risk and this rate is unacceptable to most mothers and when adequate preconception counselling is provided they will choose to withdraw from valproate and/or swap valproate for another drug. For women who have frequent seizures, the smallest dose of valproate that controls their seizures is best and polytherapy should be avoided (particularly combinations of valproate and topiramate). ${ }^{42}$ The mother should be central in our decision-making process and we should support her if, when appraised of all the evidence, she makes a decision to remain on valproate in pregnancy. She should not be made to feel selfish for considering maternal quality of life factors when making a decision. Tools for decision making, such as the optiongrids.org, are helpful when weighing up difficult choices such as these. 
We need to know how valproate causes neurodevelopmental disorders as the drug remains an important treatment for women with epilepsy. What is the risk to women with epilepsy and their children when they are unmedicated in pregnancy? When does the risk balance tip so that future advice becomes remain on your drug, or if it is valproate swap it if you can? In addition to the evidence gaps discussed above, I want to ask: has the time come for it to be compulsory to enrol all women who take valproate into a national registry? It is now clear that we should be adequately counselling every girl and women of childbearing age when valproate is first prescribed and at every subsequent review, which should be at least annually. The MHRA Toolkit would seem to be a sensible minimum standard for all who prescribe to women, and we should provide them with the 'Valproate patient guide'. Finally, it may be the time to refer to a local specialist as the MHRA advise that 'only doctors experienced in managing epilepsy (or bipolar disorder) should prescribe valproate to these patients' and that these specialists 'must supervise ongoing treatment with a review annually, at a minimum.'

\section{Conflicts of interest}

RHT is an associate editor of Practical Neurology, an associate editor of Clinical Medicine, web editor of Seizure and medical advisor to Epilepsy Professional magazine.

RHT has received honoraria from Eisai, Sanofi and UCB Pharma.

\section{Acknowledgement}

This article is based on the Linacre Lecture given at Royal College of Physicians, 13 February 2017.

\section{References}

1 Meador K, Reynolds MW, Crean S, Fahrbach K, Probst C. Pregnancy outcomes in women with epilepsy: a systematic review and metaanalysis of published pregnancy registries and cohorts. Epilepsy Res 2008:81:1-13.

2 Meador KJ, Baker GA, Browning N et al. Cognitive function at 3 years of age after fetal exposure to antiepileptic drugs. N Engl ] Med 2009;360:1597-605.

3 Harden CL, Hopp J, Ting TY et al. Practice parameter update: management issues for women with epilepsy-focus on pregnancy (an evidence-based review): obstetrical complications and change in seizure frequency: report of the Quality Standards Subcommittee and Therapeutics and Technology Assessment Subcommittee of the American Academy of Neurology and American Epilepsy Society. Neurology 2009;73:126-32.

4 Harden CL, Meador KJ, Pennell PB et al. Management issues for women with epilepsy-Focus on pregnancy (an evidence-based review): II. Teratogenesis and perinatal outcomes. Epilepsia 2009;50:1237-46.

5 Meador KJ, Baker GA, Browning $N$ et al. Fetal antiepileptic drug exposure and cognitive outcomes at age 6 years (NEAD study): a prospective observational study. Lancet Neurol 2013;12:244-52.

6 Meador KJ, Baker GA, Browning $\mathrm{N}$ et al. Breastfeeding in children of women taking antiepileptic drugs: cognitive outcomes at age 6 years. JAMA Pediatr 2014;168:729-36.

7 Adab N, Tudur Smith C, Vinten ] et al. Common antiepileptic drugs in pregnancy in women with epilepsy. Cochrane Database Syst Rev 2015;(12):CD004848.

8 Tomson T, Battino D, Bonizzoni E et al. Dose-dependent teratogenicity of valproate in mono- and polytherapy: an observational study. Neurology 2015;85:866-72.
9 Christensen J, Grønborg TK, Sørensen MJ et al. Prenatal valproate exposure and risk of autism spectrum disorders and childhood autism. JAMA 2013:309:1696-703.

10 Wood AG, Nadebaum C, Anderson V et al. Prospective assessment of autism traits in children exposed to antiepileptic drugs during pregnancy. Epilepsia 2015;56:1047-55.

11 Choi CS, Gonzales EL, Kim KC et al. The transgenerational inheritance of autism-like phenotypes in mice exposed to valproic acid during pregnancy. Sci Rep 2016;6:36250.

12 Medicines and Healthcare products Regulatory Agency. Medicines related to valproate: risk of abnormal pregnancy outcomes. London: MHRA, 2015. www.gov.uk/drug-safety-update/medicinesrelated-to-valproate-risk-of-abnormal-pregnancy-outcomes [Accessed 10 August 2017].

13 Medicines and Healthcare products Regulatory Agency. Valproate and risk of abnormal pregnancy outcomes: new communication materials. London: MHRA, 2016. www.gov.uk/drug-safety-update/ valproate-and-of-risk-of-abnormal-pregnancy-outcomes-newcommunication-materials [Accessed 10 August 2017].

14 Harden CL, Meador KJ, Pennell PB et al. Practice parameter update: management issues for women with epilepsy—focus on pregnancy (an evidence-based review): teratogenesis and perinatal outcomes: report of the Quality Standards Subcommittee and Therapeutics and Technology Assessment Subcommittee of the American Academy of Neurology and American Epilepsy Society. Neurology 2009;73:133-41.

15 Tomson T, Marson A, Boon P et al. Valproate in the treatment of epilepsy in girls and women of childbearing potential. Epilepsia 2015;56:1006-19.

16 Chen YH, Chiou HY, Lin HC, Lin HL. Affect of seizures during gestation on pregnancy outcomes in women with epilepsy. Arch Neurol 2009;66:979-84.

17 Rauchenzauner M, Ehrensberger M, Prieschl M et al. Generalized tonic-clonic seizures and antiepileptic drugs during pregnancy-a matter of importance for the baby? J Neurol 2013;260:484-8.

18 Veiby G, Daltveit AK, Engelsen BA, Gilhus NE. Pregnancy, delivery, and outcome for the child in maternal epilepsy. Epilepsia 2009;50:2130-9.

19 Man SL, Petersen I, Thompson M, Nazareth I. Antiepileptic drugs during pregnancy in primary care: a UK population based study. PLoS One 2012;7:e52339.

20 Thomas RH, Berkovic SF. The hidden genetics of epilepsy-a clinically important new paradigm. Nat Rev Neurol 2014;10:283-92.

21 Epi4K consortium, Epilepsy Phenome/Genome Project. Ultra-rare genetic variation in common epilepsies: a case-control sequencing study. Lancet Neurol 2017;16:135-43.

22 Viale L, Allotey J, Cheong-See F et al. Epilepsy in pregnancy and reproductive outcomes: a systematic review and meta-analysis. Lancet 2015;386:1845-52.

23 Perucca P, O'Brien TJ, Eadie M, Vajda FJ. Valproate still has a place in women with epilepsy. Epilepsia 2015;56:1175-6.

24 Pickrell WO, Lacey AS, Thomas RH et al. Trends in the first antiepileptic drug prescribed for epilepsy between 2000 and 2010 Seizure 2014:23:77-80.

25 Pickrell WO, Lacey AS, Bodger OG et al. Epilepsy and deprivation, a data linkage study. Epilepsia 2015;56:585-91.

26 Marson AG, Al-Kharusi AM, Alwaidh M et al. The SANAD study of effectiveness of valproate, lamotrigine, or topiramate for generalised and unclassifiable epilepsy: an unblinded randomised controlled trial. Lancet 2007:369:1016-26.

27 Edey S, Moran N, Nashef L. SUDEP and epilepsy-related mortality in pregnancy. Epilepsia 2014;55:e72-4.

28 Jones LA, Thomas RH. Sudden death in epilepsy: insights from the last 25 years. Seizure 2017;44:232-6.

29 Kapoor D, Wallace S. Trends in maternal deaths from epilepsy in the United Kingdom: a 30-year retrospective review. Obstet Med 2014;7:160-4. 
30 Perucca E. Pharmacological and therapeutic properties of valproate: a summary after 35 years of clinical experience. CNS Drugs 2002;16:695-714.

31 Löscher W. The discovery of valproate. In: Löscher W, ed. Valproate. Basel: Birkhäuser, 1999:1-3.

32 Mabunga DF, Gonzales EL, Kim JW, Kim KC, Shin CY. Exploring the validity of valproic acid animal model of autism. Exp Neurobiol 2015;24:285-300.

33 Martin HG, Manzoni OJ. Late onset deficits in synaptic plasticity in the valproic acid rat model of autism. Front Cell Neurosci 2014;8:23.

34 Walsh J, Thomas RH, Church C et al. Executive functions and psychiatric symptoms in drug-refractory juvenile myoclonic epilepsy. Epilepsy Behav 2014;35:72-7.

35 Thomas RH, Walsh J, Church C et al. A comprehensive neuropsychological description of cognition in drug-refractory juvenile myoclonic epilepsy. Epilepsy Behav 2014;36:124-9.

36 Janz D, Fuchs U. Are antiepileptic drugs harmful when given during pregnancy? Ger Med Mon 1964:9:20-2.

37 Bromfield EB, Dworetzky BA, Wyszynski DF et al. Valproate teratogenicity and epilepsy syndrome. Epilepsia 2008;49:2122-4.

38 Thomas RH, Smith PEM. Epilepsy is different. J R Soc Med 2011:104:141-3.
39 Anazi S, Maddirevula S, Faqeih E et al. Clinical genomics expands the morbid genome of intellectual disability and offers a high diagnostic yield. Mol Psychiatry 2017;22:615-24.

40 Wright CF, Fitzgerald TW, Jones WD et al. Genetic diagnosis of developmental disorders in the DDD study: a scalable analysis of genome-wide research data. Lancet 2015;385:1305-14.

41 Pennings JL, van Dartel DA, Robinson JF, Pronk TE, Piersma AH. Gene set assembly for quantitative prediction of developmental toxicity in the embryonic stem cell test. Toxicology 2011;284:6371.

42 Thomas RH, Robertson NP. The consequences of valproate exposure in utero. J Neurol 2016;263:1887-9.

43 European Medicines Agency. PRAC recommends new measures to avoid valproate exposure in pregnancy EMA/67672/2018 www.ema.europa.eu/docs/en_GB/document_library/Press release/2018/02/WC500243555.pdf [Accessed 2 March 2018]

Address for correspondence: Dr Rhys Thomas, Institute of Neuroscience, Henry Wellcome Building, The Medical School, Framlington Place, Newcastle University, Newcastle upon Tyne NE2 4HH, UK.

Email: Rhys.Thomas@ncl.ac.uk 